\title{
Commuting Regularity degree of finite semigroups
}

\section{A. FIRUZKUHY and H. DOOSTIE}

\section{ABSTRACT.}

A pair $(x, y)$ of elements $x$ and $y$ of a semigroup $S$ is said to be a commuting regular pair, if there exists an element $z \in S$ such that $x y=(y x) z(y x)$. In a finite semigroup $S$, the probability that the pair $(x, y)$ of elements of $S$ is commuting regular will be denoted by $d c r(S)$ and will be called the Commuting Regularity degree of $S$. Obviously if $S$ is a group, then $\operatorname{dcr}(S)=1$. However for a semigroup $S$, getting an upper bound for $\operatorname{dcr}(S)$ will be of interest to study and to identify the different types of non-commutative semigroups. In this paper, we calculate this probability for certain classes of finite semigroups. In this study we managed to present an interesting class of semigroups where the probability is $\frac{1}{2}$. This helps us to estimate a condition on non-commutative semigroups such that the commuting regularity of $(x, y)$ yields the commuting regularity of $(y, x)$.

\section{REFERENCES}

[1] Ahmadidelir, K., Campbell, C. M. and Doostie, H., Almost Commutative Semigroups, Algebra Colloquium, 18 (2011), 881-888

[2] Campbell, C. M., Robertson, E. F., Ruskuc, N. and Thomas, R. M., On subsemigroups of finitely presented semigroups, J. Algebra, 180 (1996), 1-21

[3] Campbell, C. M., Robertson, E. F., Ruskuc, N. and Thomas, R. M., Semigroup and group presentation, Bull. London Math. Soc., 27 (1995), 46-50

[4] Doostie, H. and Maghasedi, M., Certain classes of groups with commutativity degree $d(g)<\frac{1}{2}$, Ars combinatoria, 89 (2008), $263-270$

[5] Doostie, H. and Sadeghieh, A., On the identification of subsemigroups of transformation semigroups, J. Math. Sciences, 2 (2009), $245-256$

[6] Gustafon, W. H., What is the probability that two group elements commute?, Amer. Math. Monthly, 80 (1973), 1031-1034

[7] Howie, J., Fundamentals of semigroup theory, London Mathematical Society, New Series 12, Oxford University Press,1995

[8] Lescot, P., Isoclinism classess and commutativity degree of finite groups, J. Algebra, 177 (1995), 847-869

[9] MacHale, D., Commutativity in finite rings, Amer. Math. Monthly, 83 (1976), 30-32

[10] Robertson, E. F. and Ünlü, Y., On semigroup presentations, Proceeding of Edinburg Math. Soc., 36 (1992), 55-68

[11] Ruskuc, N., Semigroup presentation, Ph. D. Thesis (University of St. Andrews), (1995)

[12] The GAP group, GAP- Groups, Algorithms and Programming, Version 4.4, Aachen, St. Andrews, 2004 (http://www.gap-system.org)

Mathematics Department

SCICENCE AND RESEARCH BRANCH, IslamiC AZAD UNIVERSITY

P. O. BOX $14515 / 1775$, TEHRAN, IRAN

E-mail address: a zamfiruzkuhy @yahoo.com

E-mail address: doost ihegmail.com 